\title{
The Curious Case of the OZ439 Mesylate Salt: An Amphiphilic Antimalarial Drug with Diverse Solution and Solid State Structures
}

\author{
Andrew J. Clulow, ${ }^{\dagger}\left(\right.$ Malinda Salim, $^{\dagger}$ Adrian Hawley, ${ }^{\ddagger}$ Elliot P. Gilbert, ${ }^{\S}$ and Ben J. Boyd* ${ }^{\dagger}, \|($ \\ ${ }^{\dagger}$ Drug Delivery, Disposition and Dynamics, Monash Institute of Pharmaceutical Sciences, 381 Royal Parade, Parkville, Victoria 3052, \\ Australia \\ ¥Australian Synchrotron, ANSTO, 800 Blackburn Road, Clayton, Victoria 3168, Australia

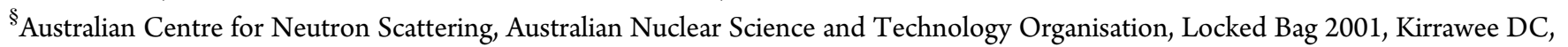 \\ New South Wales 2232, Australia \\ "ARC Centre of Excellence in Convergent Bio-Nano Science and Technology, Monash Institute of Pharmaceutical Sciences, Monash \\ University (Parkville Campus), 381 Royal Parade, Parkville, Victoria 3052, Australia
}

\section{Supporting Information}

ABSTRACT: Efforts to develop orally administered drugs tend to place an exceptional focus on aqueous solubility as this is an essential criterion for their absorption in the gastrointestinal tract. In this work we examine the solid state behavior and solubility of OZ439, a promising single-dose cure for malaria being developed as the highly water-soluble mesylate salt. The aqueous phase behavior of the OZ439 mesylate salt was determined using a combination of small angle neutron and X-ray scattering (SANS and SAXS, respectively). It was found that this salt has low solubility at low concentrations with the drug largely precipitated in free base aggregates. However, with increasing concentration these crystalline aggregates were observed to dissociate into cationic micelles and lamellar phases, effectively increasing the dissolved drug concentration. It was also found that the dissolved OZ439 spontaneously precipitated in the presence of biologically relevant anions, which we attribute to the high lattice energies of most of the salt forms of the drug. These findings show that aqueous solubility is not always what it seems in the context of amphiphilic drug molecules and highlights that its use as the principal metric in selecting drug candidates for development can be perilous.

KEYWORDS: polymorph, self-assembly, micelles, scattering, solubility

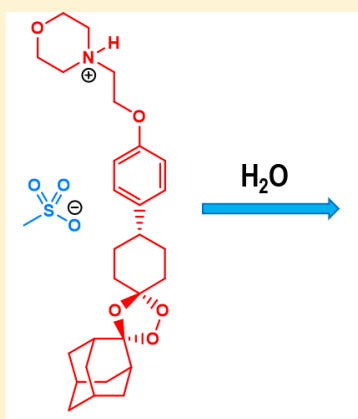

07439 mesylate

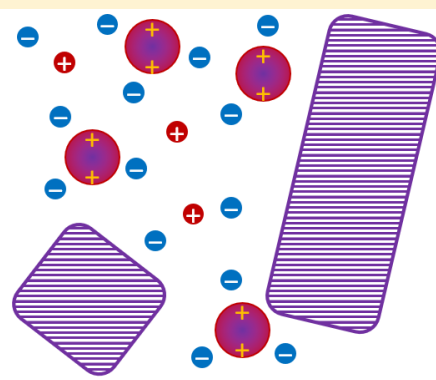

Concentration-dependent colloidal structures

\section{INTRODUCTION}

Malaria is one of the world's greatest unmet medical needs, that leads to the death of around half a million patients per year, mostly pediatric patients in tropical low economy communities. The treatment of malaria in such communities is challenging due to supply and adherence issues. Pharmacological intervention is still regarded as a key strategy to address malaria as a disease, with a strong push for new candidate drugs to which the parasite has no or limited resistance and with sufficient potency and favorable pharmacokinetic profiles to enable a single dose treatment. ${ }^{1}$

Artefenomel (OZ439) is a promising candidate against multiple malaria parasites. ${ }^{2}$ The trioxolane pharmacophore is retained from the natural antimalarial artemisinin, but the remaining structure of artefenomel is a synthetic novel structure (Figure 1). OZ439 was designed and evaluated in the early to mid-2000s specifically to extend exposure to the drug by optimization of the structure to yield favorable pharmacokinetics, ${ }^{3}$ with OZ439 displaying enhanced bioavailability in the presence of food in humans.

Although OZ439 shows outstanding pharmacokinetics and offers promise as a single dose cure for malaria, a major target population for the compound is children under the age of five for whom a conventional tablet or capsule is not considered to be an appropriate dose form. The most viable formulation approach is a liquid dose form and therefore an understanding of the behavior of OZ439 in aqueous environments in terms of solid state, dissolution, and molecular disposition in solution is critical to anticipate the impact of dispersion of a solid dose form for oral administration. The solution behavior of OZ439, which is under development in its highly soluble mesylate salt form, has not been reported.

The structure of OZ439 notably contains an ionizable polar morpholino moiety at one end of the molecule and a hydrophobic adamantyl group at the opposite end, lending

Received: February 15, 2018

Revised: $\quad$ March 15, 2018

Accepted: March 20, 2018

Published: March 20, 2018 

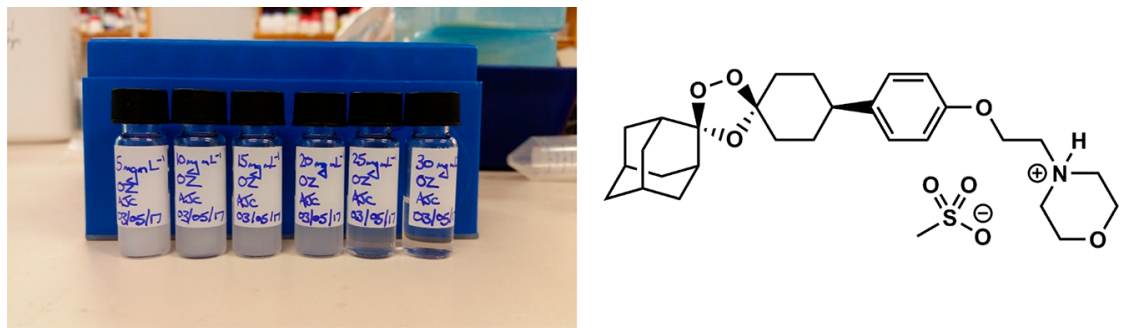

Figure 1. Image of mixtures of the OZ439 mesylate salt with $\mathrm{H}_{2} \mathrm{O}$ and the chemical structure of the OZ439 mesylate salt. The OZ439 mesylate concentration increases from left to right $\left(5,10,15,20,25\right.$, and $\left.30 \mathrm{mg} \mathrm{mL}^{-1}\right)$ illustrating the reduction in turbidity with increasing concentration.

the molecule an amphiphilic tendency. Drugs possessing an amphiphilic structure have been known to self-assemble to form micellar structures or liquid crystals, ${ }^{5}$ which can improve stability ${ }^{6}$ and have consequences for activity. ${ }^{7}$ The potential for OZ439 to self-assemble in solution therefore complicates its likely solution behavior and a thorough characterization of the phase behavior of OZ439 is of interest to both the pharmaceutical and colloid science communities. To this end, the solution and solid state phases of OZ439 mesylate were determined using a combination of small-angle X-ray and neutron scattering. Intriguingly, the OZ439 mesylate salt appears to increase in solubility with increasing concentration in simple aqueous solution, and this is related to the selfassembled phases formed by the drug. However, these colloidal structures were found to precipitate out as the hydrochloride and then the free base forms of the drug under simulated gastric and intestinal conditions, highlighting that apparent aqueous solubility alone can be a poor metric when selecting amphiphilic drugs for development in oral dosage forms.

\section{EXPERIMENTAL SECTION}

Materials. The OZ439 mesylate salt was supplied by the Medicines for Malaria Venture (MMV) and was used as received. $\mathrm{H}_{2} \mathrm{O}$ from Milli-Q water purification systems was used for all experiments, and $\mathrm{D}_{2} \mathrm{O}$ for the SANS experiments was supplied by the Australian Centre for Neutron Scattering (ANSTO, Sydney, Australia). Sodium hydroxide pellets were purchased from Merck (Darmstadt, Germany), and hydrochloric acid (36\%) was purchased from LabServ (Ireland). Both sodium hydroxide and hydrochloric acid were diluted with Milli-Q water to make solutions with concentrations between 0.1 and $2.0 \mathrm{M}$ for $\mathrm{pH}$ adjustment. Sodium chloride (USP grade) was supplied by ANSTO and was purchased from Research Organics (Cleveland, OH, USA). Unless otherwise stated all chemicals were used as received without further purification.

Small Angle Neutron Scattering (SANS). For SANS experiments, $30 \mathrm{mg} \mathrm{mL}^{-1}$ stock solutions of the OZ439 mesylate salt were prepared in mixtures of Milli-Q $\mathrm{H}_{2} \mathrm{O}$ and $\mathrm{D}_{2} \mathrm{O}(\mathrm{H} / \mathrm{D}=100: 0,50: 50,25: 75$, and $0: 100)$ supplied by ANSTO. These solutions were subsequently diluted in the same $\mathrm{H}_{2} \mathrm{O} / \mathrm{D}_{2} \mathrm{O}$ solvent mixture to give solutions with lower concentrations. Samples were mixed by vortexing immediately after preparation and were then equilibrated for at least $1 \mathrm{~h}$ before they were loaded into Hellma cells. SANS measurements were performed on the Quokka instrument at OPAL. ${ }^{8}$ Three instrument configurations were used, two with equal source-tosample and sample-to-detector distances of 20 and $8 \mathrm{~m}$, and the final configuration with a source-to-sample distance of $12 \mathrm{~m}$ and a sample-to-detector distance of $1.3 \mathrm{~m}$ with a $300 \mathrm{~mm}$ lateral detector offset to increase the maximum observable $Q$.
Source and sample aperture diameters of $50 \mathrm{~mm}$ and $12.5 \mathrm{~mm}$, respectively, were used. Neutrons with wavelength of $5 \AA(\Delta \lambda)$ $\lambda=10 \%)$ were used at all configurations. These configurations provided a continuous $Q$ range from 0.003 to $0.751 \AA^{-1}$ where $Q$ is the magnitude of the scattering vector, defined by $Q=$ $(4 \pi / \lambda) \sin (\theta)$, where $\lambda$ is the neutron wavelength and $2 \theta$ is the scattering angle. All samples were enclosed in Hellma cells with path lengths of either $1 \mathrm{~mm}(\mathrm{H} / \mathrm{D}=100: 0$ and 50:50) or $2 \mathrm{~mm}$ $(\mathrm{H} / \mathrm{D}=25: 75$ and $0: 100)$. The temperature of the samples was controlled by a Julabo thermostated bath that was held at $25^{\circ} \mathrm{C}$ throughout the measurements. All data were corrected for blocked beam measurements, normalized, radially averaged, and placed on an absolute scale, following attenuated direct beam measurements, using a package of macros in Igor Pro software (Wavemetrics, Lake Oswego, OR, U.S.A.), and modified to accept HDF5 data files from Quokka. ${ }^{9}$ The reduced data were analyzed using the SASView fitting software accounting for the experimental uncertainty in both $I(Q)$ and Q. ${ }^{10}$

Small Angle X-ray Scattering (SAXS). SAXS measurements were performed at the Small and Wide Angle X-ray Scattering (SAXS/WAXS) beamline of the Australian Synchrotron, part of ANSTO. ${ }^{11}$ The autoloader sample environment developed at the Australian Synchrotron was used for all measurements at the ambient temperature of the SAXS/WAXS experimental hutch, which is typically $27^{\circ} \mathrm{C}$. Samples were all prepared in Milli-Q $\mathrm{H}_{2} \mathrm{O}$ by dilution of four stock solutions as described in Table S1. Samples were mixed by vortexing immediately after preparation and again after 45-60 min. The samples were then left to equilibrate for at least $1 \mathrm{~h}$ before 100 $\mu \mathrm{L}$ aliquots were loaded into 96-well plates and covered with a silicone mat to prevent evaporation. The samples were drawn one at a time into a quartz capillary held stationary in the X-ray beam, and up to 13 scattering measurements were performed as the solution was drawn into and then ejected from the capillary back into the sample well. The capillary was then washed with water and $2 \%$ Helmanex detergent solution. The capillary was filled with water, and the background scattering from the waterfilled capillary was recorded to monitor capillary contamination due to beam damage prior to the next sample being measured. This was observed to be negligible for the OZ439 mesylate dispersions measured under flow. Scattering was recorded at sample-detector distances of 7159 and $1426 \mathrm{~mm}$ with a photon energy of $12 \mathrm{keV}(\lambda=1.033 \AA)$. 2D scattering patterns were radially integrated into $1 \mathrm{D}$ scattering functions $I(Q)$ using the in-house developed software package ScatterBrain. The scattering functions were put on an absolute scale with units of $\mathrm{cm}^{-1}$ using the scattering from water as a standard. The lowand high- $Q$ data were stitched together using the IRENA data analysis suite (Version 2.61) ${ }^{12}$ in the Igor Pro 7 environment and the data were analyzed using the SASView fitting 

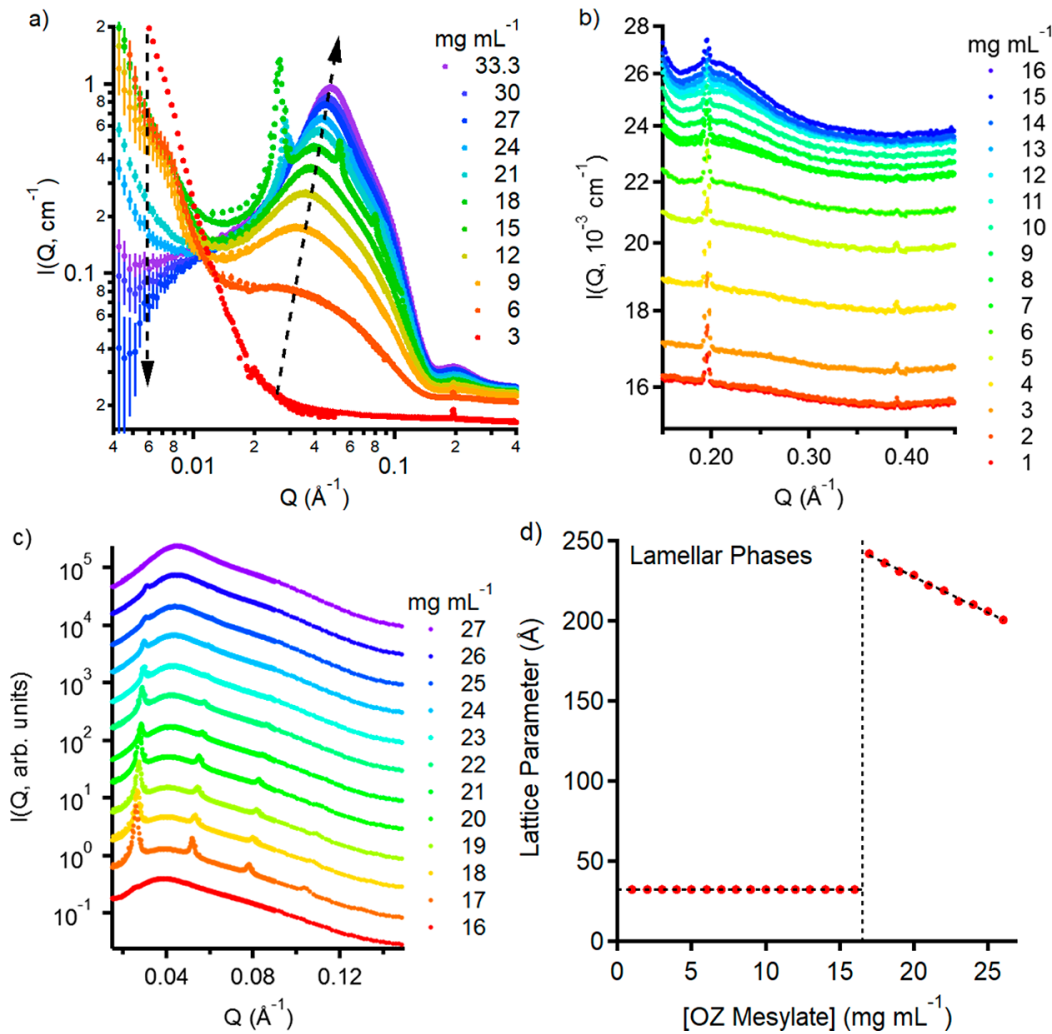

Figure 2. (a) Small angle X-ray scattering profiles of the $\mathrm{OZ} 439$ mesylate salt in $\mathrm{H}_{2} \mathrm{O}$ at concentrations from $3 \mathrm{mg} \mathrm{mL}^{-1}$ to $33.3 \mathrm{mg} \mathrm{mL}^{-1}$. Individual colored points indicate recorded data, and the dashed arrows indicate changes in intensity with increasing OZ439 mesylate concentration. Scattering intensities are on an absolute scale. (b) High- $Q$ Bragg peaks observed at low OZ439 mesylate concentrations $\left(1-16 \mathrm{mg} \mathrm{mL}^{-1}\right.$, absolute scale) and (c) low- $Q$ Bragg peaks observed in the intermediate concentration region (16-26 mg mL ${ }^{-1}$, arbitrary scale as profiles are offset for clarity). (d) Lattice parameters determined from the Bragg peaks of the OZ439 lamellar phases in panels b and c.

software $^{10}$ (Version 4.0.1). In order to model the data sets in which strong Bragg peaks overlapped with the scattering features from charged OZ439 mesylate micelles (Figure 2c), the data points relating to the Bragg peaks were removed and the remaining data points were modeled.

X-ray Diffraction Measurements on OZ439 Free Base (OZ439-FB) Powders/Dispersions. SAXS patterns of the OZ439-FB form 1 and form 2 powders were collected by loading the samples into glass microcapillaries $(1.5 \mathrm{~mm}$ outer diameter) that were placed in the X-ray beam. OZ439-FB form 1 powders were prepared from the dispersion of OZ439 mesylate in water (99 mg OZ439 mesylate in $2.5 \mathrm{~mL}$ water), and the $\mathrm{pH}$ was adjusted to $\sim 8$ using $1 \mathrm{M} \mathrm{NaOH}$ solution. The samples were vacuum filtered, and the powders were collected for SAXS measurements within $1 \mathrm{~h}$ of preparation. The OZ439FB form 1 powder was also stored at room temperature for 2 weeks, and the resultant powder (that had converted to OZ439-FB form 2) was analyzed. An X-ray beam with a photon energy of $13 \mathrm{keV}(\lambda=0.954 \AA)$ and a sample-to-detector distances between 562 and $568 \mathrm{~mm}$ were used. For dispersion measurements (Figure 5) a dispersion was placed in a thermostatted sample holder at $37{ }^{\circ} \mathrm{C}$. Dispersions were circulated through a capillary mounted in the X-ray beam using a peristaltic pump and $0.2 \mathrm{M}$ aqueous $\mathrm{NaOH}$ solution was added to the dispersion to raise the $\mathrm{pH}$.

Thermal Analysis of OZ439 Free Base Powders: SAXS. Analysis of the solid state forms of OZ439-FB form 1 powders with temperature were performed using a Mettler Toledo FP82HT hot stage with the sample window placed in the X-ray beam. OZ439-FB form 1 was prepared from an OZ439 mesylate dispersion (99 mg of OZ439 mesylate in $2.5 \mathrm{~mL}$ of water with a $\mathrm{pH}$ of $\sim 8$ adjusted using $1 \mathrm{M} \mathrm{NaOH}$ solution) and an OZ439 hydrochloride dispersion ( $99 \mathrm{mg}$ of OZ439 mesylate in $2.5 \mathrm{~mL}$ of water with a $\mathrm{pH}$ of $\sim 1$ adjusted using $1 \mathrm{M} \mathrm{HCl}$ solution). The resulting dispersions were vacuum filtered and the collected powders of OZ439-FB form 1 were transferred to a glass microscope slide covered with a glass coverslip and placed on the hot stage. The samples were heated from 30 to $120{ }^{\circ} \mathrm{C}$ with a heating rate of $5{ }^{\circ} \mathrm{C} \mathrm{min}^{-1}$. The SAXS patterns were recorded at a sample-to-detector distance of $575 \mathrm{~mm}$ using a photon energy of $13 \mathrm{keV}(\lambda=0.954 \AA)$.

Thermal Analysis of OZ439 Free Base Powders: DSC and TGA. Differential scanning calorimetry (DSC) thermograms of the OZ439-FB powders obtained from the first heating cycles were recorded on a PerkinElmer DSC 8500 (Waltham, MA, USA) between 25 and $120{ }^{\circ} \mathrm{C}$ with a heating rate of $5{ }^{\circ} \mathrm{C} \mathrm{min}^{-1}$ under nitrogen. The thermogravimetric analyses (TGA) of the OZ439-FB powders in platinum sample pans were carried out on a Pyris 1 TGA (PerkinElmer, Waltham, MA, USA) between 25 and $300{ }^{\circ} \mathrm{C}$ with a heating rate of $10{ }^{\circ} \mathrm{C} / \mathrm{min}$. The OZ439-FB powders were prepared from the OZ439 mesylate dispersion as described previously and the samples were kept in vacuum for about $2 \mathrm{~h}$ prior to the experiments.

\section{RESULTS AND DISCUSSION}

SAXS and SANS Profiles of the OZ439 Mesylate Salt in Water. The appearance of mixtures of the OZ439 mesylate salt with water $\left(\mathrm{H}_{2} \mathrm{O}\right)$ at different concentrations is shown in Figure 

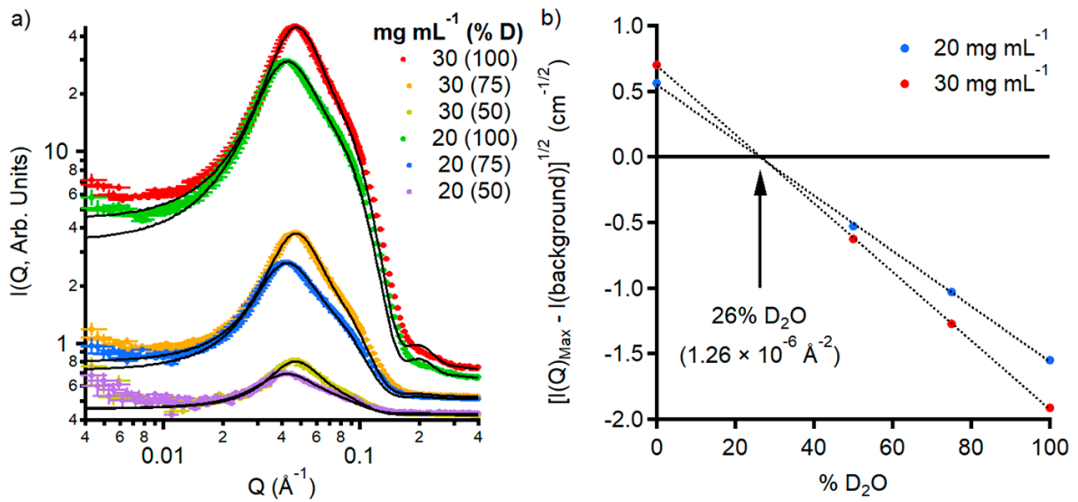

Figure 3. (a) Small angle neutron scattering profiles of the OZ439 mesylate salt in $\mathrm{H} 2 \mathrm{O} / \mathrm{D} 2 \mathrm{O}$ mixtures $(\% \mathrm{D}=50,75$, or 100) with concentrations of 20 and $30 \mathrm{mg} \mathrm{mL}^{-1}$. Individual colored points indicate recorded data and the solid black lines indicate model fits to polydisperse charged spherical particles. The intensities of $100 \% \mathrm{D}$ and $75 \% \mathrm{D}$ data have been multiplied by factors of 8 and 1.7 , respectively, for clarity. The data was placed on an absolute scale and the background scattering from the sample environment was subtracted for fitting. (b) Square root of the peak SANS intensity minus the background intensity $\left(I^{1 / 2}\right)$ versus $\% \mathrm{D}_{2} \mathrm{O}$ in the $\mathrm{H}_{2} \mathrm{O} / \mathrm{D}_{2} \mathrm{O}$ solvent. The $I^{1 / 2}$ values for 50,75 , and $100 \% \mathrm{D}_{2} \mathrm{O}$ have been ascribed negative values to allow linear fitting of the data. The contrast match point at $I^{1 / 2}=0$ is marked with the arrow.

1. The solutions were prepared by diluting a $30 \mathrm{mg} \mathrm{mL}^{-1}$ stock solution and show that as the concentration of the OZ439 salt was decreased the solutions became more turbid. This was contrary to expectation and suggested that either the salt was less soluble at lower concentrations or that large colloidal structures were forming upon dilution that led to increased light scattering. To address these hypotheses, a combined small-angle X-ray and neutron scattering (SAXS and SANS, respectively) study was performed to obtain a model for the structures of the particles that were forming as a function of OZ439 concentration.

In the X-ray study, four stock solutions were prepared with concentrations of $5,10,20$, and $33.3 \mathrm{mg} \mathrm{mL}^{-1}$ in $\mathrm{H}_{2} \mathrm{O}$. These stock solutions were diluted as indicated in Table $\mathrm{S} 1$ to afford solutions with concentrations between 1 and $30 \mathrm{mg} \mathrm{mL}^{-1}$ (1 $\mathrm{mg} \mathrm{mL} \mathrm{m}^{-1}$ increments) to generate a concentration series. Four duplicate solutions were also prepared from the different stock solutions to confirm that the structures formed were the same, which they were (Figure S1). In the corresponding neutron scattering experiments, solutions with OZ439 mesylate salt concentrations of $5,10,20$, and $30 \mathrm{mg} \mathrm{mL}^{-1}$ were prepared in $\mathrm{H}_{2} \mathrm{O} / \mathrm{D}_{2} \mathrm{O}$ mixtures with ratios of 100:0, 50:50, 25:75, and $0: 100$ to provide different solvent scattering length density (SLD) contrasts with the colloidal species forming.

Initial qualitative analysis of the SAXS profiles (Figure 2a) revealed that at low concentrations $\left(<4 \mathrm{mg} \mathrm{mL}^{-1}\right)$ only large particles were present in the solutions, as indicated by the sharp upturn in scattering intensity at low $Q$ with Bragg peaks corresponding to a lamellar phase observed at $Q=0.195$ and $0.390 \AA^{-1}$ (lattice parameter $=32.2 \AA$ ) (Figure $2 \mathrm{~b}$ ). As the concentration of the OZ439 mesylate salt increased above $4 \mathrm{mg}$ $\mathrm{mL}^{-1}$, the scattering intensity from large particles at low $Q$ became weaker and smaller particles were observed in the mixtures through the increase in scattered X-ray intensity between $Q=0.01$ and $0.30 \AA^{-1}$. The signal from these particles increased in intensity with the concentration of OZ439 mesylate salt in the solution and the downturn in scattered intensity from these smaller particles below $Q=0.03 \AA^{-1}$ combined with the profiles having weak fringes indicated that interparticle interactions were leading to measurable structure factor effects. In addition, the Bragg peaks at 0.195 and 0.390 $\AA^{-1}$ were present up to an OZ439 mesylate concentration of 16 $\mathrm{mg} \mathrm{mL}^{-1}$, above which they were no longer present but were replaced by Bragg peaks at much lower $Q$ values between 0.02 and $0.14 \AA^{-1}$ (Figure 2c). These Bragg peaks shifted to higher $Q$ values between 17 and $26 \mathrm{mg} \mathrm{mL}^{-1}$ and their intensity decreased, indicating a decrease in the lattice parameter (Figure $2 \mathrm{~d}$ ) of the lamellar phases and a commensurate decrease in concentration of the lamellar phase with increasing OZ439 mesylate concentration. Above $27 \mathrm{mg} \mathrm{mL}^{-1}$, neither Bragg peaks or low $Q$ scattering from large particles was observed in the SAXS profiles, leaving only the scattering feature from the small particles.

When the corresponding samples were prepared in $\mathrm{H}_{2} \mathrm{O}$ / $\mathrm{D}_{2} \mathrm{O}$ mixtures for the SANS measurements, it was observed that they were less turbid than the corresponding solutions prepared in $\mathrm{H}_{2} \mathrm{O}$ for the SAXS measurements. This suggested that the structure formation by the OZ439 mesylate salt might be affected by deuteration of the solvent. This was borne out in the lamellar phases observed in Figure 2c, which had larger lattice parameters (Bragg peaks at lower $Q$ ) and were observed in the SANS profiles at lower concentrations when $\mathrm{D}_{2} \mathrm{O}$ was incorporated into the solvent rather than at $20 \mathrm{mg} \mathrm{mL}^{-1}$ as they were in the SAXS profiles in $\mathrm{H}_{2} \mathrm{O}$ (Figure 3 and Figure S3). Furthermore, due to the extended duration of the SANS measurements (up to $24 \mathrm{~h}$ to complete measurements at all sample-detector distances), the larger particles had time to sediment out of the mixtures. This settling process was accompanied by the absence of the lamellar peaks at sampledetector distances recorded later in time (Figure S2), indicating that the lamellar peaks were generated by the larger particles in the mixtures that cause them to be turbid. A common feature of both the SAXS and SANS profiles (Figure 3a) was the presence of small particles presenting structure factor effects and the structures of these particles were modeled as described forthwith.

SAXS/SANS Data Modeling. The structure factor that gave reasonable fits to the scattering data in Figures 2 and 3 was the Hayter-Penfold structure factor, used to model the interactions between charged particles in solution. ${ }^{13}$ Given the miscibility of the mesylate counterions in water, this was consistent with the formation of cationic micelles containing more protonated $\mathrm{OZ} 439-\mathrm{H}^{+}$molecules than water-miscible mesylate anions. The SAXS and SANS profiles were commensurately modeled as polydisperse charged spheres (which will be referred to as either spheres or micelles) and this 
yielded the fits depicted in Figure 3 (SANS) and Figure S3 (SAXS). This fitting model had 11 fitting parameters. To reduce the number of free parameters, a number of common fitting parameters (scale factor, solvent SLD, particle SLD, temperature, dielectric constant of the solvent, ${ }^{14}$ polydispersity in radius, and background intensity) were determined and are described in Table S2 and were not allowed to vary during data modeling.

The SLD contrast match point for the OZ439- $\mathrm{H}^{+} / \mathrm{OZ} 439$ mesylate in the micelles was determined to be $26 \% \mathrm{D}_{2} \mathrm{O}$ from the peak intensity in the SANS profiles at different $\mathrm{H}_{2} \mathrm{O} / \mathrm{D}_{2} \mathrm{O}$ ratios (Figure $3 \mathrm{~b}$ ), which corresponded to a neutron scattering length density (SLD) of $1.26 \times 10^{-6} \AA^{-2}$ and a mass density of $1.31 \mathrm{~g} \mathrm{~cm}^{-3}$ for both the OZ439 mesylate salt $\left(\mathrm{C}_{29} \mathrm{H}_{43} \mathrm{NO}_{8} \mathrm{~S}\right)$ and the OZ493- $\mathrm{H}^{+}$cation $\left(\mathrm{C}_{28} \mathrm{H}_{40} \mathrm{NO}_{5}^{+}\right)$. Attempts to model the SANS data using this SLD as the sphere SLD and the SAXS data with the corresponding X-ray SLDs $\left[(12.0-12.1) \times 10^{-6}\right.$ $\left.\AA^{-2}\right]$ gave poor fits to the observed data, which suggested that the particles contained some solvent molecules. Corefinement of the SAXS and SANS data gave an optimum volume fraction of solvent $\left(\mathrm{H} / \mathrm{D}_{2} \mathrm{O}\right)$ within the particles of 0.128 , leading to average particle SLDs of 11.5 (X-ray), 1.90 (neutron, $100 \%$ $\mathrm{D}_{2} \mathrm{O}$ ), 1.68 (neutron, $75 \% \mathrm{D}_{2} \mathrm{O}$ ), and 1.46 (neutron, $50 \%$ $\left.\mathrm{D}_{2} \mathrm{O}\right) \times 10^{-6} \AA^{-2}$. These values were used as the sphere SLDs in all fits for each given solvent. Similarly, a common polydispersity in particle radius of 0.13 (ratio of the standard deviation to the mean) was found to give acceptable fits to both the SAXS and SANS data. Once the common parameters were determined, the remaining fitting parameters (salt concentration, sphere radius, charge, and volume fraction) were determined using SASView fitting software (Table 1 and Figure 4). The full SAXS data set with fitting models is given in Figure S3.

Table 1. Model Fitting Parameters for the SANS Data Shown in Figure 3

\begin{tabular}{|c|c|c|c|c|c|}
\hline sample & $\begin{array}{l}\text { sphere } \\
\text { charge }\end{array}$ & $\begin{array}{c}\text { sphere } \\
\text { radius } \\
(\AA)\end{array}$ & $\begin{array}{l}\text { sphere } \\
\text { volume } \\
\text { fraction }\end{array}$ & $\begin{array}{l}{[\text { salt }]} \\
(\mathrm{mM})\end{array}$ & $\begin{array}{c}\text { fraction of } \\
\text { OZ439 } \\
\text { dissolved }^{a}\end{array}$ \\
\hline $\begin{array}{c}30 \mathrm{mg} \mathrm{mL}^{-1} \\
(100 \% \mathrm{D})\end{array}$ & 25 & 26.5 & 0.0235 & 0.9 & 0.94 \\
\hline $\begin{array}{c}30 \mathrm{mg} \mathrm{mL}^{-1} \\
(75 \% \mathrm{D})\end{array}$ & 24 & 26.5 & 0.0236 & 0.7 & 0.94 \\
\hline $\begin{array}{c}30 \mathrm{mg} \mathrm{mL}^{-1} \\
(50 \% \mathrm{D})\end{array}$ & 23 & 27.0 & 0.0236 & 0.9 & 0.95 \\
\hline $\begin{array}{c}20 \mathrm{mg} \mathrm{mL}^{-1} \\
(100 \% \mathrm{D})\end{array}$ & 22 & 26.5 & 0.0155 & 1.1 & 0.95 \\
\hline $\begin{array}{c}20 \mathrm{mg} \mathrm{mL}^{-1} \\
(75 \% \mathrm{D})\end{array}$ & 21 & 26.4 & 0.0155 & 1.0 & 0.94 \\
\hline $\begin{array}{c}20 \mathrm{mg} \mathrm{mL}^{-1} \\
(50 \% \mathrm{D})\end{array}$ & 19 & 26.8 & 0.0161 & 1.0 & 0.97 \\
\hline
\end{tabular}

${ }^{a}$ Represents the fraction of OZ439 molecules either incorporated into charged micelles or in dissolved salt form.

Initially it was assumed that the solution had an ionic strength of zero (salt concentration $=0 \mathrm{mM}$ ) and the optimum values of micelle charge were determined. The models produced revealed a steadily increasing micelle charge with OZ439 concentration but were nonoptimal as they did not accurately reproduce the low- $Q$ downturn in scattered intensity, particularly where it was more clearly observed at higher OZ439 mesylate concentrations containing few large particles. When the fits were performed, while allowing both the salt concentration and micelle charge to be free parameters, a steady increase in micelle charge was still observed but there was no clear trend in the behavior of the dissolved salt concentration, which had an average and standard deviation of $4.9 \pm 1.5 \mathrm{mM}$ across the concentration range studied. As no other salts had been added, it was deemed that the nonzero salt concentration must result from an increase in the concentration of OZ439 mesylate dissolved in the bulk solution at concentrations on the order of millimolar. Attempts to control the salt concentration by adding sodium chloride led to precipitation of the OZ439 micelles, presumably as the highly insoluble hydrochloride salt and this will be discussed in more detail in a later section. The SAXS data could be satisfactorily modeled in each case using the average salt concentration of 4.9 $\mathrm{mM}$, and this was used throughout the SAXS modeling.

It was found from the SAXS data that the volume fraction of micelles increased linearly with OZ439 mesylate concentration above $3.9 \mathrm{mg} \mathrm{mL}^{-1}$ (Figure $4 \mathrm{a}$ ), which is the apparent critical micelle concentration (CMC) of the system. This is an apparent CMC because not all of the OZ439 mesylate added is in the form of fully dissolved salt at this concentration. Below the apparent $\mathrm{CMC}$ the $\mathrm{pH}$ of the solutions was observed to decrease from around 3.1 to around 2.6, and above it a $\mathrm{pH}$ of $2.58 \pm 0.05$ was observed for all dispersions (Figure 4a). As the concentration of OZ439 mesylate salt was increased, the charge on the spherical micelles was found to increase (Figure $4 \mathrm{~b}$ ) and so was the radius/aggregation number (which includes OZ439 in both cationic and salt forms) of the micelles (Figure 4c), which may be related to the increased electrostatic repulsion due to having more OZ439- $\mathrm{H}^{+}$groups lacking their mesylate counterions in the micelles. The fraction of dissolved OZ439 molecules in micelles and present in the bulk as dissolved salt was calculated as described in the Supporting Information and is shown in Figure 4d. It was found that as the concentration of OZ439 mesylate was increased that the fraction of OZ439 molecules (either incorporated into charged micelles or dissolved as free salt) rose to be 1.0 at the concentrations at which the solutions become visually transparent. It was therefore posited that the remaining OZ439 in the lower concentration solutions was dispersed in large particles with lamellar structures, and the nature of these particles will be discussed in a later section in the context of the solid state behavior of OZ439 salts.

When the same model was applied to the SANS profiles of OZ439 mesylate dispersions recorded in $\mathrm{H}_{2} \mathrm{O} / \mathrm{D}_{2} \mathrm{O}$, similar trends were observed but the distribution of the OZ439 between micelles and dissolved salt was found to be different in the deuterated solvents. Only the 20 and $30 \mathrm{mg} \mathrm{mL}^{-1}$ data were modeled in the higher contrast solvents as the other SANS profiles either had weak intensity over the incoherent background scattering (particularly the samples in $\mathrm{H}_{2} \mathrm{O}$ ) or had lamellar peaks of variable intensity due to sedimentation that would interfere with the analysis (Figure S2). The volume fraction of micelles determined from the SANS profiles of solutions containing $\mathrm{D}_{2} \mathrm{O}$ (Table 1 ) was slightly higher than that determined from the corresponding SAXS profiles in $\mathrm{H}_{2} \mathrm{O}$ (Figure 4a), and the concentrations of dissolved salt were commensurately lower, being $0.9 \pm 0.1 \mathrm{mM}$. This difference is likely due to differing solubility of the OZ439 mesylate salt in $\mathrm{D}_{2} \mathrm{O}$, which was observed visually in the difference in turbidity of the dispersions containing $\mathrm{D}_{2} \mathrm{O}$. Overall, the concentrations of dissolved OZ439 were similar in the $\mathrm{H}_{2} \mathrm{O} / \mathrm{D}_{2} \mathrm{O}$ solvent mixtures, being between 0.94 and 0.97 at the high OZ439 mesylate concentrations. 

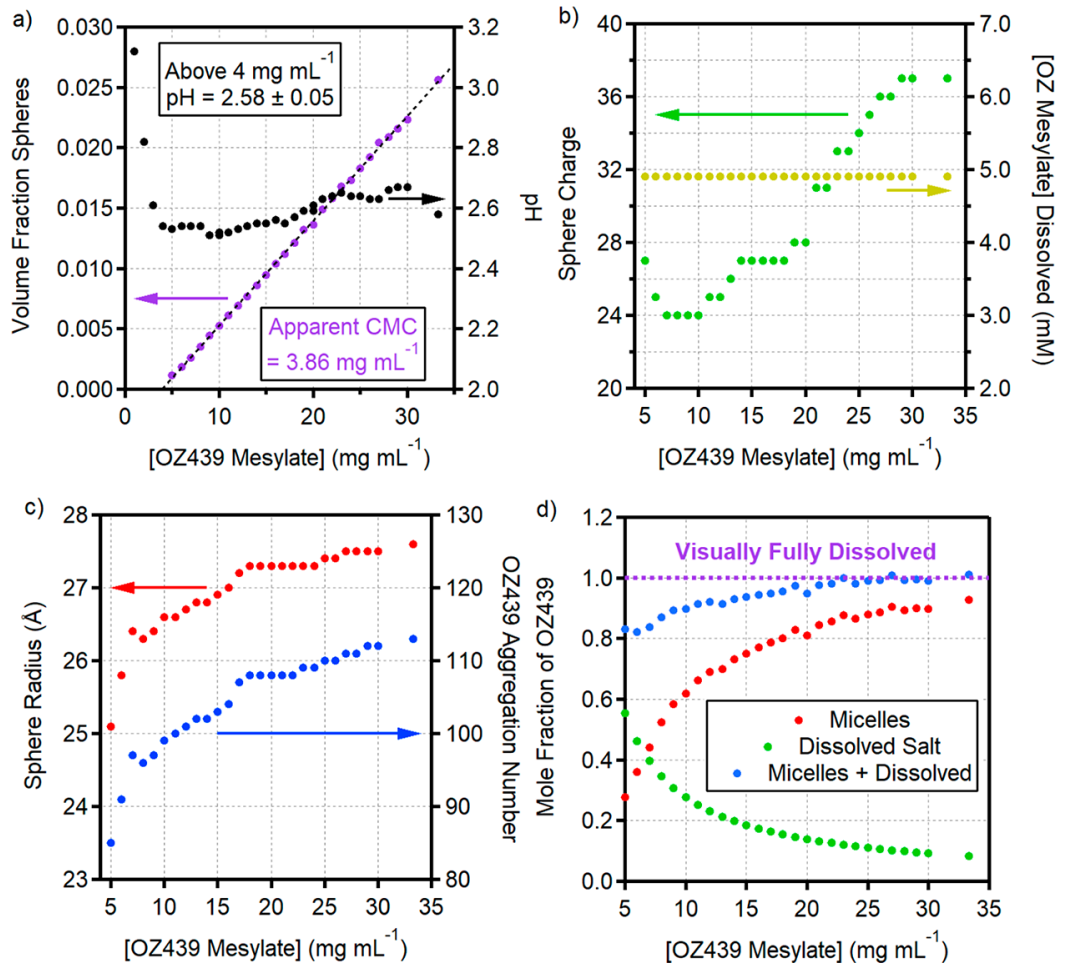

Figure 4. Model parameters determined from the SAXS analysis of OZ439 mesylate/water mixtures. (a) Sphere radius and OZ439 aggregation number, (b) sphere charge and salt concentration, and (c) sphere volume fraction determined by the SASView fitting software from the SAXS profiles. (d) Mole fraction of OZ439 molecules present as dissolved salt and in charged micelles as a function of OZ439 mesylate concentration.

Precipitation of the OZ439 Hydrochloride Salt and Formation of Free Base Polymorphs. When taken orally, the OZ439 mesylate salt passes through the stomach on its way to the intestines, where the majority of drug absorption should occur. This exposes the drug to acidic conditions rich in chloride ions. In the current study, as mentioned earlier, when aqueous OZ439 mesylate solutions were exposed to sodium chloride, precipitation of the component micelles occurred. To study the precipitation behavior of the OZ439 mesylate salt and simulate passage through the gastrointestinal tract, dispersions of the OZ439 mesylate salt in water were diluted using $10 \mathrm{vol} \%$ of $0.1 \mathrm{M}$ hydrochloric acid solution, and the SAXS profile of the dispersion was recorded as $0.2 \mathrm{M}$ sodium hydroxide solution was added to raise the $\mathrm{pH}$ (Figure 5).

$\mathrm{X}$-ray diffractograms of the OZ439 hydrochloride dispersion are shown in Figure 5 with $\mathrm{pH}$ ranging from 1.87 to 10.20 . As the $\mathrm{pH}$ was raised above 2.8 , the $\mathrm{OZ} 439$ hydrochloride salt transformed into a free base (FB) polymorph, which we have named form 1 for reasons that shall become clear. The characteristic Bragg peaks of OZ439-FB form 1 were confirmed separately by isolation of its powder from both OZ439 mesylate and OZ439 hydrochloride dispersions at pH 6.5 (Figure 6a). The FB form 1 diffractograms possessed four Bragg peaks corresponding to a lamellar phase in the range $0.19 \leq Q \leq 0.80$ (Figure 6b), the first two of which correlate with the lamellar peaks observed in the SAXS profiles of OZ439 mesylate dispersions (Figure $2 \mathrm{~b}$ ). This suggests that the large particles observed in the SAXS experiments that make low concentration $\left(<17 \mathrm{mg} \mathrm{mL}{ }^{-1}\right)$ OZ439 mesylate dispersions turbid are composed of OZ439-FB form 1 .

An independent solid-solid structural transformation of isolated OZ439-FB form 1 powder occurred during 10 days of storage at room temperature into a more thermodynamically

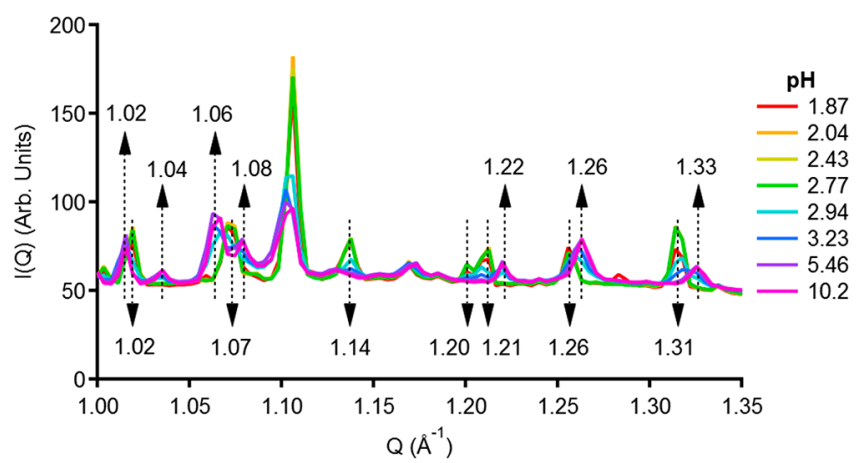

Figure 5. X-ray diffractograms of OZ439 hydrochloride in water during titration with $\mathrm{NaOH}$ solution beginning with $\mathrm{OZ} 439-\mathrm{HCl}$ at $\mathrm{pH}$ 1.87. Changes in the diffractograms were observed with increasing $\mathrm{pH}$, as the OZ439 hydrochloride salt transitioned to a free base polymorph (FB form 1). The dashed arrows indicate which peaks decrease in intensity (belonging to OZ439 hydrochloride) and which peaks increase in intensity (belonging to OZ439-FB form 1 ) as the $\mathrm{pH}$ of the suspension was increased. The background intensity from water in the capillary used for the measurements has been subtracted from all data sets.

stable form OZ439-FB form 2. Prominent and characteristic Bragg peaks of OZ439-FB form 2 at $Q=1.27$ and $1.35 \AA^{-1}$ were found to appear, with the disappearance of the prominent OZ439-FB form 1 peak at $Q=1.31 \AA^{-1}$ (Figure 6b). To elucidate whether OZ439-FB form 2 was a polymorph or a different hydrate form, thermal analyses of the compound using thermal gravimetric analysis (TGA) and differential scanning calorimetry (DSC) were conducted.

DSC analysis of the OZ439-FB form 1 and 2 powders (Figure $7 \mathrm{a}$ ) suggested that both powders were indeed free base forms of OZ439 and not a salt form, indicated by the 

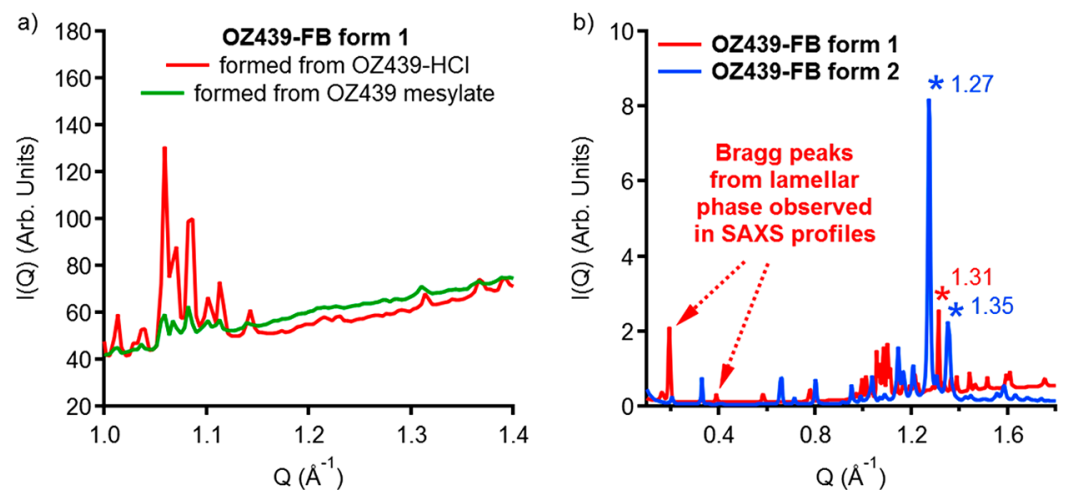

Figure 6. (a) X-ray diffractograms of OZ439-FB form 1 powders precipitated from OZ439 hydrochloride (red) and OZ439 mesylate (green) dispersions in water by $\mathrm{pH}$ adjustment to 6.5. (b) Comparison between the X-ray diffractograms of OZ439-FB form 1 powder freshly prepared from an OZ439 mesylate solution at $\mathrm{pH} 6.5$ and the same powder after 10 days of storage at room temperature to form OZ439-FB form 2. The asterisks indicate prominent characteristic peaks allowing the identification of OZ439-FB form 1 (red) and form 2 (blue).
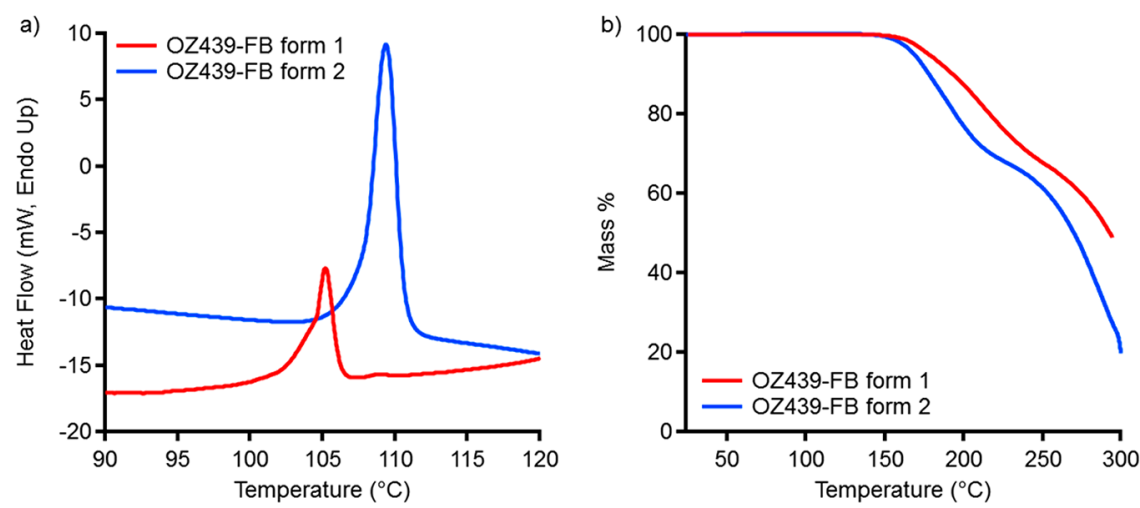

Figure 7. (a) DSC thermograms indicating the melting ranges of crystalline OZ439-FB form 1 and OZ439-FB form 2. The peak melting temperatures of OZ439-FB form 1 and form 2 were around 105 and $109{ }^{\circ} \mathrm{C}$, respectively. (b) The thermogravimetric weight loss profiles of OZ439FB form 2 as a function of temperature.

occurrence of the melting peak at low temperatures around 100-115 ${ }^{\circ} \mathrm{C}$. DSC thermograms for the OZ439 mesylate and hydrochloride salts did not reveal a melting transition below the observed onset of decomposition at around $160{ }^{\circ} \mathrm{C}$ (data not shown). The observed melting temperature of OZ439-FB form 2 was slightly higher than that of OZ439-FB form 1, suggesting that OZ439-FB form 2 was more thermally stable. No significant mass loss was observed in the TGA thermograms of either OZ439-FB form 1 or form 2 below $140{ }^{\circ} \mathrm{C}$, which was around $30{ }^{\circ} \mathrm{C}$ above their melting points and indicates that both were anhydrous solid forms of the OZ439 free base.

Although the free base forms of the OZ439 were characterized separately by XRD prior to the DSC measurements, changes in the polymorphic form of the metastable FB form 1 during heating also occurred. In situ monitoring of the crystallinity of OZ439-FB form 1 was performed using a hot stage with the sample window placed in the X-ray beam of the SAXS/WAXS beamline at the Australian Synchrotron. OZ439FB form 1 powder was freshly prepared from an OZ439 hydrochloride dispersion as described in the Experimental Section. The X-ray diffractograms in Figure 8 show that OZ439-FB form 1 (prepared from the hydrochloride salt) transformed into form 2 on heating, supporting the hypothesis that FB form 2 is a more thermodynamically stable polymorph than FB form 1 .

The Combined Picture. Based on the combined SAXS/ SANS analysis of the solution phase behavior and the

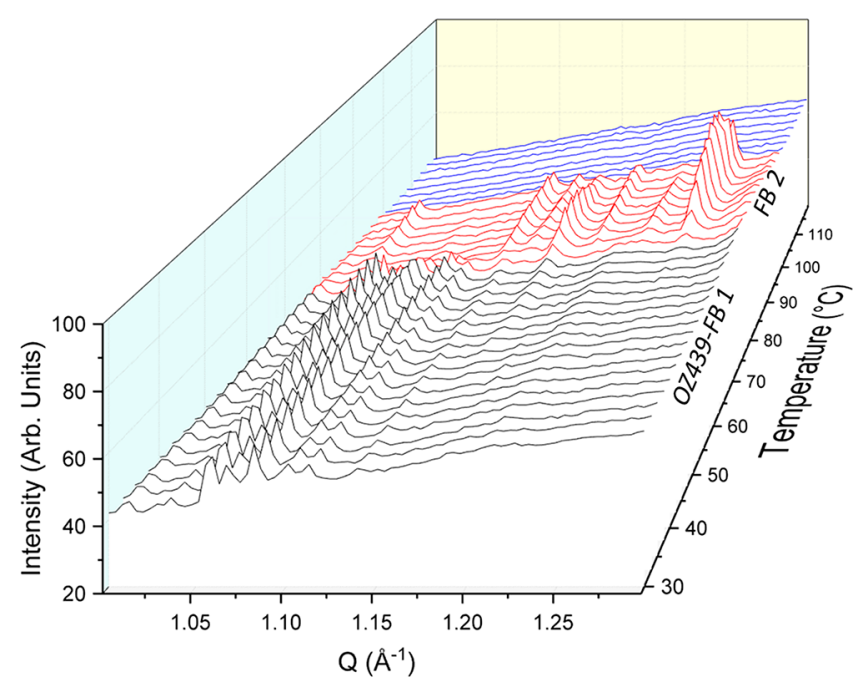

Figure 8. X-ray diffractograms OZ439-FB form 1 as a function of temperature. The FB form 1 transforms into FB form 2, which subsequently transforms to the isotropic melt.

diffractometry analysis of the precipitated powders, the sequence of structural transformations of OZ439 mesylate in solution can be inferred (Scheme 1). At low concentrations $(<4$ $\mathrm{mg} \mathrm{mL} \mathrm{m}^{-1}$ ) where $\mathrm{pH}$ is not especially low, the OZ439 mesylate salt disperses in water as large aggregates of free base form 1 
Scheme 1. Proposed Phase Transitions of the OZ439 Mesylate Salt as a Function of Concentration in Water, Determined Using a Combination of Solution Small Angle Neutron and X-ray Scattering Data and Powder X-ray Diffractometry ${ }^{a}$

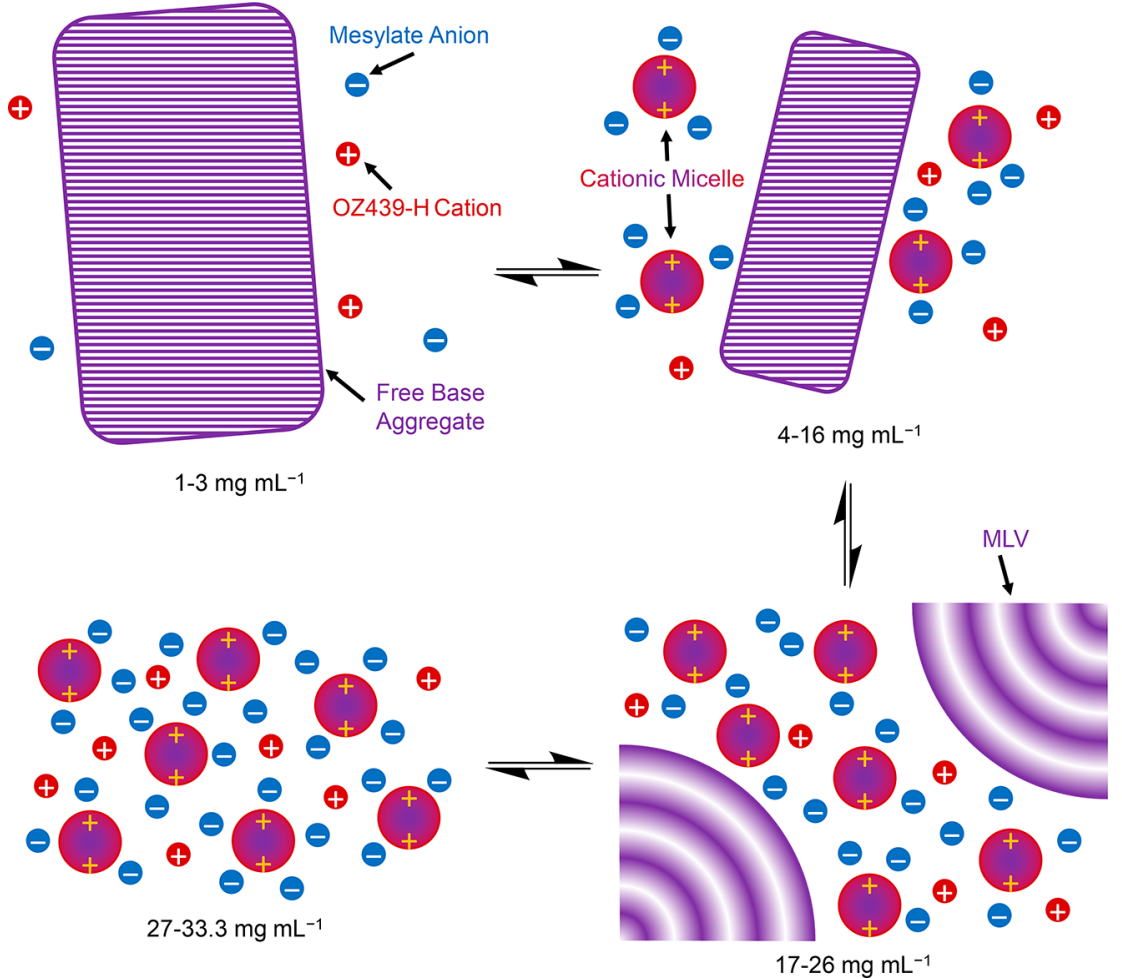

${ }^{a} \mathrm{MLV}=$ multilamellar vesicle; the individual structures are not drawn to scale.

with a fraction of dissolved OZ439 mesylate salt on the order of $<10 \mathrm{mM}$. Above the apparent CMC at $3.9 \mathrm{mg} \mathrm{mL} \mathrm{m}^{-1}$, charged micelles begin to form in solution among the free base aggregates and dissolved salt, with the volume fraction of micelles increasing in proportion to the amount of OZ439 mesylate thereafter. As the concentration of OZ439 mesylate increases, less of the salt added is dispersed as large free base aggregates, and at $17 \mathrm{mg} \mathrm{mL} \mathrm{m}^{-1}$ the aggregates undergo a structural transformation to a species with a much larger lattice parameter (Figure 2d). Given the large lattice parameter of the aggregates above $17 \mathrm{mg} \mathrm{mL}^{-1}$, it is hypothesized that these are multilamellar vesicles that are converted to micelles as the concentration of OZ439 mesylate is further increased. Above $27 \mathrm{mg} \mathrm{mL}^{-1}$, only the charged micelles and dissolved salt are resolved by the SAXS and SANS measurements, and at this point the solutions are no longer turbid but become translucent with almost all of the OZ439 mesylate dissolved either as micelles or as free salt in the bulk solution.

Upon the addition of hydrochloric acid, the OZ439 precipitates out as the highly insoluble OZ439 hydrochloride salt and this would be expected to happen in the stomach after oral administration. This phenomenon has not been reported in the academic literature but is cited as the motivation for a clinical trial in which the free powder of the drug was compared to an enteric-coated capsule on the basis that hydrochloride formation was leading to variable bioavailability. ${ }^{15}$

The hydrochloride salt is converted to a free base form upon raising the $\mathrm{pH}$ to near neutral levels as would be the case in the intestine. The free base initially formed (form 1) is thermodynamically unstable with respect to a second polymorph (form 2) to which it can be converted by heating or by storage at room temperature. These insoluble hydro- chloride and free base forms are therefore expected to dominate the species present in vivo and therefore are the primary forms of OZ439 immediately prior to absorption of the active drug.

Thus, in considering future formulation approaches for the delivery of OZ439, the interactions of the free base form with exogenous formulation components need to be considered. The poorly soluble, hydrophobic nature of the free base forms of OZ439 predicate that a lipid formulation approach may be of utility in enhancing oral delivery of this drug and in clinical trials the absorption of OZ439 has been shown to be promoted by the presence of food. In forthcoming studies we will interrogate the behavior of OZ439 in the presence of lipidcontaining formulations and study the behavior of the drug during the digestion process to better enlighten the nature of this food effect.

\section{CONCLUSION}

It has been shown that the antimalarial drug OZ439 displays a rich variety of structural behavior both in solution and in the solid state. In solution, the drug forms a concentrationdependent mixture of dissolved salt, charged micelles, free base aggregates, and potentially multilamellar vesicles. When these mixtures were exposed to biorelevant conditions reflective of the gastrointestinal tract, the drug precipitated due to the extreme insolubility of many salts of OZ439. The OZ439 hydrochloride salt formed under simulated gastric conditions can be converted to a free base form by raising the $\mathrm{pH}$ to intestinal levels. A slow transformation of the free base formed initially (form 1) to a more thermodynamically stable form (form 2) occurred upon aging the free base at room temperature and upon heating. The premise that improving the aqueous solubility of a poorly water-soluble drug by 
incorporating it into a salt form is well established but selfassembly and subsequent interactions with endogenous salts and environments of changing $\mathrm{pH}$ are also vital determinants of intestinal solubility, as clearly shown by the case of OZ439. To understand the absorption of this drug after oral administration, it appears pertinent to focus on the solubility of the free base polymorphs of OZ439 in the preabsorptive intestinal environment and not the initially soluble mesylate salt when designing formulations for OZ439 administration.

\section{ASSOCIATED CONTENT}

\section{S Supporting Information}

The Supporting Information is available free of charge on the ACS Publications website at DOI: 10.1021/acs.molpharmaceut.8b00173.

Dilution strategy for OZ439 mesylate solutions used in the SAXS measurements, raw SANS data, common fitting parameters for scattering data modeling, the full SAXS data set for OZ439 mesylate/water mixtures with model fits, and the calculation used to quantify dissolved drug (PDF)

\section{AUTHOR INFORMATION}

\section{Corresponding Author}

* Monash Institute of Pharmaceutical Sciences, Monash University (Parkville Campus), 381 Royal Parade, Parkville, VIC 3052, Australia. Tel: +61 3 99039112. Fax: +61 3 99039583. E-mail: ben.boyd@monash.edu.

\section{ORCID}

Andrew J. Clulow: 0000-0003-2037-853X

Ben J. Boyd: 0000-0001-5434-590X

\section{Notes}

The authors declare no competing financial interest.

\section{ACKNOWLEDGMENTS}

This work was funded by the Bill and Melinda Gates Foundation Grant No. OPP1160404, in collaboration with the Medicines for Malaria Venture (MMV). Funding is also acknowledged from the Australian Research Council under the Discovery Projects scheme DP160102906. We acknowledge the support of the Australian Centre for Neutron Scattering, Australian Nuclear Science and Technology Organisation (ANSTO), in providing the neutron research facilities used in this work and for travel support. The SAXS experiments for this work were conducted on the SAXS/WAXS beamline of the Australian Synchrotron, part of ANSTO. The authors thank Prof. Susan Charman, Dr. Niya Bowers, and Dr. Hanu Ramachandruni for important technical and historical discussions around OZ439, and Dr. Wye-Khay Fong for assistance with DSC and TGA measurements.

\section{REFERENCES}

(1) Mischlinger, J.; Agnandji, S. T.; Ramharter, M. Single dose treatment of malaria - current status and perspectives. Expert Rev. AntiInfect. Ther. 2016, 14 (7), 669-678.

(2) Phyo, A. P.; Jittamala, P.; Nosten, F. H.; Pukrittayakamee, S.; Imwong, M.; White, N. J.; Duparc, S.; Macintyre, F.; Baker, M.; Möhrle, J. J. Antimalarial activity of artefenomel (OZ439), a novel synthetic antimalarial endoperoxide, in patients with Plasmodium falciparum and Plasmodium vivax malaria: an open-label phase 2 trial. Lancet Infect. Dis. 2016, 16 (1), 61-69.
(3) Charman, S. A.; Arbe-Barnes, S.; Bathurst, I. C.; Brun, R.; Campbell, M.; Charman, W. N.; Chiu, F. C. K.; Chollet, J.; Craft, J. C.; Creek, D. J.; Dong, Y.; Matile, H.; Maurer, M.; Morizzi, J.; Nguyen, T.; Papastogiannidis, P.; Scheurer, C.; Shackleford, D. M.; Sriraghavan, K.; Stingelin, L.; Tang, Y.; Urwyler, H.; Wang, X.; White, K. L.; Wittlin, S.; Zhou, L.; Vennerstrom, J. L. Synthetic ozonide drug candidate OZ439 offers new hope for a single-dose cure of uncomplicated malaria. Proc. Natl. Acad. Sci. U. S. A. 2011, 108 (11), 4400-4405.

(4) Moehrle, J. J.; Duparc, S.; Siethoff, C.; van Giersbergen, P. L. M.; Craft, J. C.; Arbe-Barnes, S.; Charman, S. A.; Gutierrez, M.; Wittlin, S.; Vennerstrom, J. L. First-in-man safety and pharmacokinetics of synthetic ozonide OZ439 demonstrates an improved exposure profile relative to other peroxide antimalarials. Br. J. Clin. Pharmacol. 2013, 75 (2), 535-548.

(5) Bunjes, H.; Rades, T. Thermotropic liquid crystalline drugs. J. Pharm. Pharmacol. 2005, 57 (7), 807-816.

(6) Wallace, S. J.; Li, J.; Nation, R. L.; Prankerd, R. J.; Velkov, T.; Boyd, B. J. Self-Assembly Behavior of Colistin and Its Prodrug Colistin Methanesulfonate: Implications for Solution Stability and Solubilization. J. Phys. Chem. B 2010, 114 (14), 4836-4840.

(7) Shackleford, D. M.; Prankerd, R. J.; Scanlon, M. J.; Charman, W. N. Self-Micellization of Gemfibrozil 1-O- $\beta$ Acyl Glucuronide in Aqueous Solution. Pharm. Res. 2003, 20 (3), 465-470.

(8) Gilbert, E. P.; Schulz, J. C.; Noakes, T. J. 'Quokka'-the smallangle neutron scattering instrument at OPAL. Phys. B 2006, 385-386 (Part 2), 1180-1182.

(9) Kline, S. Reduction and analysis of SANS and USANS data using IGOR Pro. J. Appl. Crystallogr. 2006, 39 (6), 895-900.

(10) Doucet, M.; et al. SasView version 4.0; http://www.sasview.org/ and DOI: $10.5281 /$ zenodo.159083.

(11) Kirby, N. M.; Mudie, S. T.; Hawley, A. M.; Cookson, D. J.; Mertens, H. D. T.; Cowieson, N.; Samardzic-Boban, V. A lowbackground-intensity focusing small-angle $\mathrm{X}$-ray scattering undulator beamline. J. Appl. Crystallogr. 2013, 46 (6), 1670-1680.

(12) Ilavsky, J.; Jemian, P. R. Irena: tool suite for modeling and analysis of small-angle scattering. J. Appl. Crystallogr. 2009, 42 (2), 347-353.

(13) Hayter, J. B.; Penfold, J. An analytic structure factor for macroion solutions. Mol. Phys. 1981, 42 (1), 109-118.

(14) Vidulich, G. A.; Evans, D. F.; Kay, R. L. The Dielectric Constant of Water and Heavy Water between 0 and $40^{\circ}$. J. Phys. Chem. 1967, 71 (3), 656-662.

(15) https://clinicaltrials.gov/ct2/show/NCT01732588. 